\title{
Une approche comparative des discours institutionnels en éducation aux médias : une analyse socio-anthropologique
}

A comparative approach to institutional discourses on media education. A socioanthropological analysis

\section{Marlène Loicq}

\section{(2) OpenEdition Journals}

Édition électronique

URL : http://journals.openedition.org/aad/2420

DOI : $10.4000 /$ aad. 2420

ISSN : 1565-8961

Éditeur

Université de Tel-Aviv

\section{Référence électronique}

Marlène Loicq, « Une approche comparative des discours institutionnels en éducation aux médias une analyse socio-anthropologique », Argumentation et Analyse du Discours [En ligne], 19 | 2017, mis en ligne le 15 octobre 2017, consulté le 23 septembre 2019. URL : http://journals.openedition.org/aad/ 2420 ; DOI : 10.4000/aad.2420

Ce document a été généré automatiquement le 23 septembre 2019

\section{cc) (†) $\ominus$}

Argumentation \& analyse du discours est mis à disposition selon les termes de la licence Creative Commons Attribution - Pas d'Utilisation Commerciale - Pas de Modification 4.0 International. 


\title{
Une approche comparative des discours institutionnels en éducation aux médias : une analyse socio-anthropologique
}

\author{
A comparative approach to institutional discourses on media education. A socio- \\ anthropological analysis
}

Marlène Loicq

\section{Introduction}

1 Depuis leur apparition, les médias ont suscité à la fois espoirs et craintes, et l'innovation technologique a eu une résonance particulière dans le domaine éducatif. Chargée de la transmission des savoirs légitimes, de la socialisation et de l'acculturation des jeunes, l'école s'est souvent sentie menacée par la présence accrue des médias. Dans un même temps, l'introduction des problématiques médiatiques dans les débats sur l'éducation et sur les modalités d'apprentissage a encouragé le renouvellement des questionnements sur les fonctions de l'école. Le débat quant à la place à accorder aux médias à l'école est soutenu par différents arguments : celui de l'attrait de la dimension technologique - voir le débat sur les nouvelles technologies de l'information et de la communication NTIC (Barats 2007) et les technologies de l'information et de la communication pour l'enseignement - TICE, (Paquelin 2009, Perraya 2011) - et des possibilités d'innovation pédagogique et didactique permises par ces NTIC (Moeglin 2005, Cerisier 2014); celui de la dimension esthétique et culturelle de l'image (La Borderie 1997, Boutin 2011) versus la nécessité de se prémunir de l'éventualité de contenus inappropriés (violents ou pornographiques par exemple - Jehel 2000, FrauMeigs 2011); enfin, celui de former de futurs citoyens de sociétés hautement médiatisées, impliquant le développement d'un esprit critique (Piette 1996, Gonnet 1995). 
2 L'intégration des médias à l'école a globalement suivi l'évolution de leur implantation dans la vie sociale (Carlsson et al., 2008). Fondée sur des théories et des modèles élaborés dans le champ de la recherche en communication, information et médias (Anderson 1980, Masterman et Mariet 1994, Piette 1988, Loicq 2011, 2012, Landry et Bégin 2016), "l'éducation aux médias" fait aujourd'hui l'objet d'un consensus international encouragé par des organismes tels que l'UNESCO ou la Commission Européenne. Depuis 2012 et la "Déclaration de Moscou », on parle également d'«éducation aux médias et à l'information" (EMI) (traduction faite de Media and Information Literacy, $\mathrm{MIL}^{2}$ ), laquelle est récemment intégrée aux curriculums français.

3 L'éducation aux médias et à l'information résulte de stratégies internationales de reconnaissance et de préconisation, en même temps que de logiques nationales singulières lisibles dans les programmes scolaires culturellement très marqués. L'éducation aux médias comme projet est conçue au niveau international à partir des propriétés générales des médias mais au niveau des politiques éducatives. Elle reste cependant dépendante des situations locales - comme le montre la comparaison des discours institutionnels de notre corpus. L'étude comparative des cas de l'Australie, du Québec et de la France que nous avons menée propose de faire apparaître selon une approche socio-anthropologique l'importance du contexte.

\section{L'analyse socio-anthropologique de discours institutionnels}

4 L'analyse socio-anthropologique de discours institutionnels s'appuie sur le croisement de l'analyse d'éléments formels (entretiens avec les acteurs du domaine, analyse des interdiscours et cotextes) et non formels (immersion, dynamiques sociales et historiques perçues in situ) qui aident à l'interprétation de cette mise en mots officielle. En d'autres termes, l'ensemble des discours ici étudiés permet de saisir la possibilité de ce projet dans le cadre éducatif qui l'accueille et le contraint en même temps. Si cette approche est probante pour saisir l'activité discursive des institutions, elle l'est d'autant plus dans une posture comparatiste qui permet de distinguer les éléments propres à l'objet de ceux inhérents au contexte. C'est dans la dynamique de similarité et de différence que s'offrent les caractéristiques des discours.

\subsection{L'étude de cas en comparaison internationale}

Pour déterminer les terrains de notre étude, c'est-à-dire désigner « les situations ou les lieux empiriques où le chercheur va collecter des données, construire ses corpus » (Charaudeau, Maingueneau 2002 : 568), nous avons adopté une démarche d'immersion qui visait à saisir le phénomène dans son contexte et les diverses dynamiques qui le traversent. Selon une perspective comparative internationale, nous avons dû circonscrire ces terrains dans des ensembles suffisamment cohérents pour être comparables. Nous avons pour cela opté pour l'étude de cas, c'est-à-dire la «technique particulière de cueillette, de mise en forme et de traitement de l'information qui cherche à rendre compte du caractère évolutif et complexe des phénomènes concernant un système social comportant ses propres dynamiques » (Mucchielli 2004 : 92). Elle est un « plan de recherche complet en soi » (Karsenti et Demers $2000: 226)$. 
6 Cette méthode nous a permis de circonscrire les "cohérences nationales" d'un système social spécifique lesquelles sont mises à l'épreuve de la comparaison internationale telle que la préconise Maurice dans son approche sociétale :

La comparabilité ne s'applique pas dans ce cas directement à des phénomènes (ou à des objets) particuliers comparés terme à terme, elle s'applique plutôt à des ensembles de phénomènes qui constituent dans leurs interdépendances des « cohérences » nationales, propres à chaque pays. [...] «Acteurs » et «espaces» sont saisis plutôt comme autant de « construits sociaux » dont l'approche sociétale révélera dans un premier temps la spécificité, c'est-à-dire la discontinuité d'un pays à l'autre (1989: 182-184).

7 Pour saisir toute la dynamique de ces phénomènes, nous avons opté pour une immersion de longue durée car, comme le souligne Clanet (1985), c'est par les déplacements symbolique, spatial et/ou temporel que le questionnement culturel prend toute sa mesure. En allant "à la recherche des mémoires perdues », et en nous servant de l'autre culture comme "révélateur » de la nôtre, nous avons construit des données particulièrement probantes (Loicq 2011).

\subsection{De la constitution du corpus de discours institutionnels}

8 Le corpus ainsi constitué a permis de faire la genèse de l'éducation aux médias et de prendre en compte les « traces communicationnelles » (Bardin $2003: 247$ ) soumises aux procédures analytiques. Le corpus principal est composé des discours officiels et des données recueillies lors de l'immersion (éléments relevés dans les interdiscours, discours "provoqués» par des entretiens ${ }^{3}$ avec les acteurs du domaine étudié). Ce corpus secondaire vise à compléter, interpréter, expliquer le corpus principal.

9 L'analyse des discours institutionnels telle que nous la concevons, associée aux entretiens des acteurs et aux données contextualisantes, relève de ce que oger et Ollivier-Yaniv (2006: 64) nomment l'« anthropologie des discours institutionnels ».

10 Les discours institutionnels sur l'éducation aux médias en Australie ${ }^{4}$, au Québec et en France sont alors considérés comme les documents fondateurs à cette institutionnalisation de l'éducation aux médias qui, étudiés au regard du contexte qui les a produits, sont aussi le reflet des situations sociales du moment. Ce sont des expressions des idéologies et des connaissances officiellement acceptées sur le domaine, créditées de la légitimité d'énonciation des "formations discursives" (Foucault 1969) et dépendantes de ses "conditions historiques de production» (Bonnafous et Tournier, 1995). Notre corpus présente donc ce qu' " est » l'éducation aux médias officielle pour chacun des cas étudiés, et nous y analysons ce qu'ils donnent plus largement à voir de la société à laquelle ils participent. Ainsi, pour reprendre les mots de Salazar-Orvig (2003: 271), on dira que l'analyse du discours s'apparente à la compréhension de « la parole des acteurs sociaux [...], de ce que ces dires véhiculent, ce qu'ils représentent ou ce qu'ils donnent à voir ".

11 En partageant l'expérience des acteurs de l'institutionnalisation du domaine, croisée avec les données documentaires annexes (rapports, notes, colloques, etc.), nous avons pu retracer la genèse de l'éducation aux médias de nos trois terrains et ainsi restituer les « références endogènes » dans lesquelles le discours prend sens. 


\subsection{Une histoire en trois étapes, une énonciation à deux voix} grandes étapes auxquelles correspondent trois registres: la phase militante durant laquelle les discours partagés sont diffus, peu ou pas archivés ; la phase d'organisation sociale durant laquelle les acteurs se structurent et les discours se construisent et s'harmonisent; et la phase d'institutionnalisation des discours à partir d'acteurs identifiés et légitimés. Les temporalités sont complexes et imbriquées. La phase d'institutionnalisation est différée (1978/1981 en Australie, 2004/2006 au Québec, 2006/2016 en France) et se mettra en place de façon très distincte selon les cas. Nous les détaillerons par la suite.

ont donc dans un premier temps été portés par des groupes militants (éducateurs, parents d'élèves, téléspectateurs, travailleurs sociaux, etc.) autour d'un support privilégié (cinéma en Australie, télévision au Québec et presse écrite en France) et généralement en réponse à une préoccupation voire une inquiétude face à l'avènement des médias dans la vie sociale. (problématique de décrochage scolaire en Australie, réactions à des événements sociaux tragiques au Québec, mouvements d'éducation populaire en France). Le langage mobilisé et les arguments avancés sont alors porteurs de valeurs émancipatrices. médias un enjeu social et elles tissent alors des liens avec les décideurs politiques. Le processus d'institutionnalisation ainsi enclenché permet une visibilité accrue du domaine et sa reconnaissance politique. Cela conduit notamment à une prescription ministérielle. histoire dans l'évolution des formations discursives. La phase militante est incontestablement structurante dans l'aboutissement politique de l'éducation aux médias mais elle a laissé assez peu de traces concrètes. Nous avons cependant pu la prendre en compte dans notre analyse grâce aux récits des éducateurs pionniers.

La reconnaissance de deux instances permet de suivre le processus d'institutionnalisation. La première des instances «légitimes" est à l'origine de ressources pédagogiques et de discours programmatiques ( $\mathrm{ATOM}^{5}$ en Australie, le CREM $^{6}$ au Québec et le CLEMI ${ }^{7}$ en France). La seconde, prescriptive, pose les injonctions à « éduquer aux médias ». Si dans nos trois cas on distingue deux sources d'énonciation, les relations qu'elles entretiennent entre elles diffèrent.

Dans le cas de l'Australie, l'Australian Teachers of Media (ATOM), rassemble les enseignants, organise des rencontres, produit des ressources et publie deux magazines. Association totalement indépendante de l'instance institutionnelle, les acteurs d'ATOM ont néanmoins participé à l'écriture et à la publicisation du premier cours d'éducation aux médias qui prendra place dans les programmes du Victorian Institute of Secondary Education en $1978^{\circ}$. Il y a trouvé une place pérenne dès lors qu'il a été intégré au Victorian Curriculum and Assessment Authority (VCAA) et pris sa place dans les Victorian Essential Learning Standards et le Victorian Certificate of Education comme matière à crédit (comptabilisée pour le passage à l'université). ATOM et le Victorian Minister of Education

Argumentation et Analyse du Discours, 19 | 2017 
ont alors toujours été complémentaires mais autonomes, la première accompagnant de son expertise le second tout en conservant sa liberté critique. Cette collaboration perdure puisqu'ils se sont ensuite engagés conjointement dans l'écriture d'un programme national (Dunscombe et Loicq 2012). Cette dynamique conduit à un enseignement bien implanté dans les écoles et bénéficiant d'un accompagnement complet (en termes de moyens et de contenus), à savoir un projet qui fonctionne dans la logique scolaire.

19 Au Québec, le Centre de Ressources en Education aux Médias (CREM) est né en 1998 dans le sillon des états-généraux de l'éducation qui, dès 1995, et à la suite de l'importante réforme de l'éducation engagée dans les années 1960, se sont donné pour mission de définir les objectifs et missions de l'école du 21è siècle. Accompagnée de plusieurs rapports fondateurs, cette réforme a donné lieu, en 2001, à la création du programme de formation de l'école québécoise. Le CREM participe alors à l'organisation de rencontres visant à réunir les connaissances et ressources nécessaires pour proposer un cours d'éducation aux médias qui, au sein de ce programme, se cristallisera dans le module "médias» intégré parmi les domaines généraux de formation, mis en application en 2006. Le Ministère de l'Éducation, des Loisirs et du Sport (MELS) a encouragé la création du CREM et bénéficié de ses services, bien que celui-ci ait toujours cherché à garder une forme d'autonomie, notamment en se donnant pour mission de développer la lecture et le jugement critique à l'égard des informations médiatiques, non seulement des élèves mais aussi du grand public. Plus officiellement, le CREM avait comme objectif d'accompagner l'intégration des pratiques pédagogiques du module «médias » dans le programme. Cependant, après l'institutionnalisation du domaine, le MELS n'a pas renouvelé son soutien au CREM qui, malgré sa fonction de formation des enseignants et de développement de projets et de recherches, a cessé d'exister dès 2005, faute de moyens et à cause de diverses discordances en son sein. Les relations entre ces deux instances sont donc inégales et ambigües, voire conflictuelles. Cette éducation aux médias se retrouve alors privée du soutien de toute instance programmatique (le CREM, pour les contenus et la formation des enseignants) ou prescriptive (le MELS, personne n'ayant officiellement la responsabilité du dossier « médias » au sein du ministère).

En France, le Centre pour l'éducation aux médias et à l'information, est officiellement chargé depuis 1983 de «l'éducation aux médias dans l'ensemble du système éducatif français ». Il est placé sous tutelle du Ministère de l'éducation nationale (MEN). Malgré le travail important du CLEMI dans le domaine depuis les années 80, le MEN n'intègrera explicitement l'éducation aux médias dans les programmes scolaires obligatoires qu'avec la création en $2006 \mathrm{du}$ "Socle commun des connaissances et des compétences », issu de la « loi d'orientation et de programme pour l'avenir de l'école » promulguée en $2005^{\circ}$. Elle prendra ensuite une place concrète dans les programmes avec la création du parcours citoyen et en réponse aux vagues d'attentats connues en France en 2015. La création des «Enseignements pratiques interdisciplinaires » (EPI) et de l'«Enseignement Moral et Civique " (EMC) se présentent ainsi comme des cadres privilégiés pour mettre en œuvre cette éducation aux médias que le CLEMI a la charge assumée et revendiquée d'accompagner. Aujourd'hui, la restructuration des instances éducatives et des réseaux ("Canopé », "Direction Numérique Éducatif », etc.) vient sûrement changer les dynamiques discursives et les demandes implicites d'expertise ${ }^{10}$. 
21 Dans chacun des cas, les deux instances ont leur histoire propre mais, confrontées à la nécessité de former les jeunes dans des sociétés hautement médiatisées, elles envisagent les médias, les élèves et l'école parfois de façon très différente. Ce sont précisément ces tensions que fait apparaitre la comparaison. En effet, les instances internationales tendent à donner l'illusion d'une vision homogène, transversale et donc finalement aculturelle de l'éducation aux médias alors que sa mise en œuvre dans les programmes scolaires nationaux montre d'importantes divergences.

\section{Contextualisation des discours institutionnels de l'éducation aux médias}

22 À la dualité de ces instances fait écho la dualité des discours. D'un côté nous envisageons les discours programmatiques des instances légitimes comme autant de délimitations de ce que le domaine inclut et exclut, en y associant valeurs et normes. Nous les appréhendons alors comme des discours institutionnels « dont la pluralité renvoie à des situations d'énonciation variables relativement à l'institution - et comportant notamment des positions «semi-officielles » (Oger et Ollivier-Yaniv 2006: 63). De l'autre, sont pris en compte les discours prescriptifs de l'instance institutionnelle, c'est-à-dire les documents officiels du programme d'éducation dans lesquels est introduit le cours d'éducation aux médias, ceux qui donnent sens aux actes de la collectivité en justifiant la pratique sociale et en la délimitant. Considérés comme pouvant servir de garants aux autres discours, ils pourraient être constituants (Maingueneau et Cossutta 1995). Appréhendés dans le cadre restreint de l'institution scolaire et d'un programme à instituer, portés par une visée performative, nous les considérons dès lors comme des discours instituants. Ces deux types de discours contribuent à faire fonctionner l'éducation aux médias dans le système social (scolaire, éducatif) et les imaginaires, à établir de manière plus ou moins contraignante la réalité sociale qu'ils énoncent.

\subsection{L'Australie ou la transparence des discours}

En Australie, l'éducation aux médias a été intégrée très tôt dans les curricula. Aussi, particulièrement pour ce cas, comprendre le processus d'institutionnalisation passe d'abord par l'histoire de la transformation de pratiques isolées en discours prescriptifs, puis par l'évolution de ces discours au sein des politiques éducatives. Cette histoire, racontée à notre demande ${ }^{11}$ par quelques acteurs de ce processus, fait écho à l'étude diachronique des programmes édités de 1986 à 2011 dans le Study Design du VCAA, mais aussi dans les ressources produites par ATOM, notamment les guides Media 1\&2. Intégré au curriculum des Arts pour sa pédagogie du « faire » (produire une émission radio, un court métrage, etc.), l'enseignement en lien avec les médias a été peu à peu étayé par une approche théorique d'analyse des discours médiatiques, des institutions, de l'économie, des publics. Il visait initialement les élèves en plus grandes difficultés scolaires, et a été plébiscité pour l'aspect innovant d'un projet ludique permettant de répondre à la politique du maintien des élèves en classe. Au fil des ans, le cours s'est ouvert à l'ensemble des profils étudiants et a même été pris en compte dans la certification de fin d'études. Ces étapes sont lisibles dès les plus anciens programmes d'éducation aux médias, notamment dans l'argumentaire concernant la 
complémentarité des approches pratique et théorique, l'intérêt qu'il représente dans une perspective professionnelle mais aussi plus largement citoyenne.

La lecture diachronique de ces discours instituants témoigne de l'ouverture de l'éducation aux médias à l'intégration de la dimension pédagogique et du degré de précision toujours plus grand des connaissances sur les médias que cela implique. Dès le premier programme étudié ${ }^{12}$, aucune formule argumentative n'est présente, seule l'utilisation de la forme passive concernant les objectifs à atteindre par le programme laisse présager de la nouveauté du projet. Le caractère prescriptif du document s'affirme avec l'introduction de verbes à l'infinitif ou au présent simple, et à travers la précision apportée à l'objet étudié. Les médias sont alors présentés comme objet social (place, effets, fonctions) et comme objet d'apprentissage à partir notamment de leurs fonctions (informer, communiquer, connecter, divertir, éduquer...), de leurs effets (influencer, construire, jouer un rôle, donner forme, contribuer...), de leur nature (diverse, dynamique, évolutive, créative...), de leur fonctionnement (industrie, structures politiques, pratiques professionnelles, technologies...) et de leurs structures (codes et conventions, formes, textes, genres, langages...). Les médias y sont également associés à une dynamique communicationnelle dont les fonctions sont largement inscrites dans l'histoire de cet immense continent isolé qui a tiré un bénéfice important du développement des moyens de communication pour connecter chaque région entre elles et au reste du monde.

Comme il est attendu dans le cadre de l'étude de discours instituants, les marques d'énonciation sont quasi-absentes de ces textes, signe d'une prise de distance avec le public cible du texte (enseignants), le projet (élèves) et l'objet médias (publics) alors que les discours institutionnels d'ATOM associent les médias, dans des nominations inclusives, aux enseignants et aux élèves. Ces discours se réclament d'ailleurs d'une légitimité qui se construit par référence externe, étant donné l'omniprésence des médias et la nécessité de répondre aux attentes éducatives australiennes; et interne, étant donné l'engagement d'experts dans la production des dits documents.

Ces discours (instituants et institutionnels) semblent entretenir une relation de complémentarité, chacun d'eux étant suffisamment riche pour répondre aux attentes définitoires du projet, l'un ayant le pouvoir de prescrire et l'autre, du fait de son autonomie critique, d'inscrire le projet dans un ensemble de normes et de valeurs socio-historiques particulières à l'Australie ${ }^{13}$.

\subsection{Le Québec ou l'influence de deux modèles}

Pour comprendre les discours instituants de l'éducation aux médias au Québec, il est nécessaire de prendre en considération les rapports fondateurs issus des différentes réformes, dont celui du "Groupe de Travail sur la Réforme du Curriculum » (GTRC 1997). Le document s'inscrit lui-même dans « l'adaptation du curriculum d'études aux changements sociaux", parmi lesquels la diversité sociale et culturelle d'un Québec ancré dans une mondialisation avancée. Les médias y sont présentés comme "dispositifs techniques » servant à la " mise en circulation massive de l'information ». On souligne leur rôle dans le développement de la personnalité des jeunes, voire dans la fabrique de l'opinion et l'on affirme la nécessité d'intégrer ce module comme "domaine d'expériences de vie ", étant donné que « l'école ne peut faire abstraction de l'influence exercée par les médias sur les enfants et les adolescents, acteurs de la 
démocratie de demain ». Dans le texte prescriptif issu de ce rapport, nous retrouvons une courte présentation des médias, de leurs fonctions (" constituent des modes d'accès à ») et de leurs effets («influencent et parfois déterminent... contribuent à façonner... »), toujours envisagés dans leur « relation à » (l'individu, la société, l'école). On constate que la formule «éducation aux médias » disparaît au profit de la simple mention "médias». Les énoncés prescriptifs présentent un vocabulaire riche et varié en lien avec la démarche éducative, et assez peu descriptif. La forme passive ou les formules comme "l'école propose ", " est conviée à jouer un rôle » ou les élèves sont " invités à » ou " amenés à » sont privilégiées comme l'utilisation de verbes au futur ou au conditionnel. Les axes de développement du projet sont présentés sous formes nominales (et non verbales) et renvoient à des dispositions mentales plutôt qu'à des verbes d'action. Ainsi, les discours instituants ne remplissent pas totalement leur fonction prescriptive mais sont plutôt de l'ordre de l'incitatif.

Les discours institutionnels font, quant à eux, état de nombreuses marques d'énonciations (« les médias façonnent nos attitudes, nos opinions tout en constituant une source importante où nous puisons abondamment les sujets qui alimentent nos échanges et nos conversations avec nos proches " - je souligne) renvoyant le lecteur à son univers familier (" dès le réveil, nos radios d'auto, les écouteurs de nos baladeurs, le soir quand nous revenons chez nous »). L'implication de l'acteur, individuel ou collectif, à travers laquelle se construit la première relation aux médias, puis l'éducation aux médias, est sans cesse rappelée, pour souligner que par les médias, l'enfant « ne fait pas que se divertir, il apprend sur lui-même et sur le monde ». Dans ce projet, l'acquisition de connaissances sur les médias est secondaire au regard de l'aspect proprement éducatif du projet. La légitimité de ce discours, et à travers lui, du projet d'éduquer aux médias, s'appuie d'abord sur une légitimité projetée en impliquant le lecteur dans la détermination de la nécessité de ce projet ("chacun est à même de constater ", " cela relève désormais du cliché », "nul besoin d'insister »), puis par une légitimité externe passant par une institution qui en reconnaît l'importance. La fonction normative de ce texte est alors le symptôme de la nécessité toujours présente d'encourager, voire de convaincre de l'utilité d'une éducation aux médias.

29 L'institutionnalisation de l'éducation aux médias au Québec a largement été accompagnée par les acteurs du CLEMI (réseau français) et leur l'influence est perceptible. Cependant, au Québec, les notions introduites ne renvoient pas aux mêmes réalités. Les discours programmatiques du CREM, comme en témoigne le document d'accompagnement de l'intégration de cet enseignement dans les pratiques scolaires, incitent à une éducation à la critique de l'information médiatique. Mais celle-ci y est appréhendée comme inhérente au fonctionnement médiatique (les médias sont des discours, en cela ils transmettent de l'information), et non pas comme genre (l'information d'actualité), comme c'est le cas en France. De même, la dimension citoyenne importée du CLEMI ne renvoie pas à la même réalité au Québec où elle est associée à la pluralité culturelle articulée à l'usage de la langue française. Entre l'influence du modèle français et les apports théoriques post-modernes américains, le Québec propose une réponse singulière aux changements sociaux entamés dans les années 1980.

30 Finalement, au Québec, les discours instituants et institutionnels semblent indépendants les uns des autres. Cependant, ils placent unanimement l'élève au centre, en vue de l'inculcation d'un "savoir-être ». L'école, concurrencée par les médias, doit 
pourtant les apprivoiser pour être plus proche de la réalité de l'élève. C'est donc la nécessaire collaboration de l'école et des médias qui se dessine dans ce projet éducatif.

\subsection{La France ou l'incohérence narrative qui subsiste}

31 En France, si la création du CLEMI marque l'institutionnalisation de l'éducation aux médias, son inscription dans les programmes scolaires n'apparaît que récemment. Pourtant, forte de son existence officielle depuis 1982, on pourrait s'attendre à une évolution progressive des discours officiels, plutôt qu'à ce que l'on peut rapprocher des "évènements discursifs » (Moirand 2007) épars et ponctuels qui se présentent comme autant d'étapes d'officialisation. Par ailleurs, l'analyse des différents énoncés révèle de nombreux paradoxes à la fois dans les contenus et les logiques discursives. L'argument présent de 1976 à 2011 pour une " modernisation de l'école » perd son sens dès lors que celui-ci est associé à l'idée que l'éducation aux médias ne doit en aucun cas modifier le projet de l'école et qu'il ne sera pas nécessaire de "sortir des savoirs que l'école est chargée de transmettre, ajouter des activités, s'aventurer sur des territoires mal explorés». Aussi, notons le paradoxe présent dans le rapport annexé à la loi d'orientation et des programmes (2005) qui préconise de «renforcer » l'éducation aux médias alors que celle-ci n'apparait nulle part dans les programmes précédents. Par ailleurs, le discours instituant est largement ancré dans l'idée de performance (" accomplir avec succès, construire, réussir, participation efficace et constructive ", etc.). Or ce champ lexical n'est pas utilisé pour définir les actions de l'élève, mais bien celles de l'institution. C'est bien encore un discours pour convaincre le MEN du bienfondé de l'éducation aux médias que porte le CLEMI. Les discours institutionnels semblent quant à eux manquer leur fonction prescriptive puisque l'unique mention faite à l'éducation aux médias l'est sous l'emploi de la forme passive (« être éduqué aux médias»). Cette mention n'est par ailleurs accompagnée d'aucune autre précision, faisant ainsi implicitement un renvoi de compétence définitoire et programmatique vers l'instance légitime (le CLEMI). Les discours institutionnels prennent donc en charge toute la tâche de dessiner le projet, notamment dans les guides pédagogiques « éduquer aux médias, ça s'apprend! » destinés aux enseignants.

Dans la droite ligne de l'interdiscours, les médias sont présentés comme des « agitateurs " que l'école doit apprivoiser en vue de former des citoyens éclairés. Ils sont présentés comme des «filtres » qui font «écran aux connaissances ", dont il est nécessaire d'acquérir un "usage sûr et raisonné des différents supports et des différents contenus $»^{14}$. L'emploi récurrent d'adjectifs comme "indispensable», "fondamental » et des indications temporelles justifient l'urgence et participent à donner à ces documents une fonction argumentative plutôt que véritablement programmatique. Aussi, nous sommes plus dans le registre du "possibilitif » que dans celui du performatif. Nous retrouvons cette dynamique au sein des logiques de légitimation externe (référence à l'adoption du projet par les instances nationales, européennes et internationales) et traditionnelle (les piliers de l'école sont solides). L'école est donc porteuse d'un imaginaire sinon hostile, du moins frappé de scepticisme à l'encontre des médias et de la légitimité des pratiques. Les marques d'énonciation ne sont pas dirigées vers l'interlocuteur réel (l'enseignant), mais toujours dans une démarche argumentative visant à faire accepter la pertinence d'une éducation aux médias à l'institution. Curieuse relation interlocutive qui se perpétue du premier guide de 2006 à celui de 2011. 
Ces discours montrent une relation d'interdépendance. Les discours prescriptifs utilisent la formule "éducation aux médias " sans même envisager de la définir, renvoyant à l'instance légitime (CLEMI) la fonction de lui donner corps (fonction programmatique). Mais celle-ci est toujours dans un discours militant argumentatif visant notamment à convaincre le MEN de la nécessité de mettre en place ce domaine à l'école. Faire de l'éducation aux médias, c'est d'abord répondre aux attentes de l'école de la république, c'est la moderniser sans pour autant la modifier. Garante des savoirs légitimes et partagés, elle se sent donc concurrencée par les médias qu'il lui faut mettre au service de l'idéologie républicaine.

\begin{tabular}{|l|l|l|l|}
\hline & AUSTRALIE & QUEBEC & FRANCE \\
\hline Fonction des médias & $\begin{array}{l}\text { Médias comme } \\
\text { médiation }\end{array}$ & Médias comme outils & $\begin{array}{l}\text { Médias comme filtre à la } \\
\text { connaissance }\end{array}$ \\
\hline $\begin{array}{l}\text { Relation de l'école aux } \\
\text { médias }\end{array}$ & Reconnaissance & Collaboration & Transformation \\
\hline Approche éducative & $\begin{array}{l}\text { Projets } \\
\text { communication }\end{array}$ & Projets d'éducation & Projets citoyens \\
\hline Enjeux pour l'école & Opportunité & Nécessité & Défi \\
\hline Approche des médias & Potentiel éducatif & Potentiel éducatif & Pas de mention \\
caractère éducatif
\end{tabular}

Tableau récapitulatif des principales caractéristiques de l'éducation aux médias dans les trois contextes étudiés

\section{Conclusion}

L'articulation des discours instituants et institutionnels permet de circonscrire « ce qui doit être dit et fait » en éducation aux médias, à la fois dans sa forme la plus scolaire, prescrite par l'institution, et dans ses formulations plus normatives, portées par les instances légitimes. Ainsi, pour comprendre le projet d'éducation aux médias, il faut se saisir des discours historiques et officiels en contexte puisque l'éducation aux médias apparaît dépendante, d'une part, d'une histoire sociale de relations avec les médias et donc des imaginaires qui en découlent; d'autre part, de l'appropriation des connaissances sur les médias produites par les disciplines scientifiques concernées par les institutions; et enfin, de la capacité de ces institutions à mettre en mots ces 
relations souhaitées entre l'école, l'élève et les médias. Ces analyses discursives ont alors confirmé l'hypothèse issue de l'immersion, à savoir que l'éducation aux médias fonctionne en tant que sujet, elle est «intégrée " comme cours au curriculum en Australie; elle doit être mise en place comme projet "autonome» dans les programmes québécois; elle demande à être intégrée de manière "transversale » en France en n'étant pas un cours mais un élément à associer aux enseignements disciplinaires.

Aussi, ce sont finalement les lieux même de compréhension de ces discours qui diffèrent d'un cas à l'autre puisqu'en Australie, c'est dans les politiques éducatives que l'étude de cas a pu trouver le plus d'éléments à sa constitution, étant donné que l'éducation aux médias y a très tôt été institutionnalisée et transformée en programme dont les contenus théoriques et pratiques ont pu être interprétés grâce à la rencontre des acteurs de ces discours officiels. Ce sont alors les textes prescriptifs qui se sont avérés les plus riches. Au Québec, ce sont les préoccupations sociales qui ont fait émerger le domaine et c'est à travers elles que l'on saisit l'essence du projet. Les discours présentent une éducation aux médias largement centrée sur l'élève et ses expériences de vie, tout comme les différents aspects culturels et sociaux de cette province tentant de suivre une idéologie largement ancrée dans le social. C'est donc par l'immersion et l'ouverture du corpus à tous les travaux annexes aux documents officiels que l'on peut réellement saisir le sens de cette éducation aux médias officielle mais en marge. Ce sont les rapports accompagnant les réformes qui se sont avérés les plus parlants. En France, les textes officiels ne trouvent de sens que dans la reconstitution de l'histoire qui les accompagne. En effet, les formes discursives de l'éducation aux médias dans les programmes scolaires restent largement informatives (plutôt que prescriptives) et appellent à un complément d'information que seules d'autres instances peuvent donner. Autrement dit, en France, l'éducation aux médias (qui doit être transversale aux disciplines) ne "se dit " pas précisément dans les programmes. Son destinataire, l'enseignant, doit lui aussi reconstituer un parcours définitionnel pour donner de la profondeur et du sens à ce qui lui est sommé d'enseigner.

Reconstituer l'histoire d'un domaine, ce n'est pas seulement relever le récit de ceux qui y ont participé, bien que cela soit indispensable, c'est aussi saisir les idéaux et les imaginaires qui, pour un temps, ont guidé les actions, des plus activistes aux plus officielles. Voilà pourquoi il a été nécessaire, dans cette recherche, d'articuler les discours institutionnels avec les situations sociales, qui se révèlent être en écho des imaginaires sociaux nationaux. C'est ainsi que ces discours officiels se sont présentés comme largement contraints par les modèles socio-politiques dans lesquels ils prenaient place. Les caractéristiques du multiculturalisme australien se sont lues dans ces discours centrés sur les médias et qui mettent l'emphase sur la question de la pluralité du sens. L'idéologie interculturelle du Québec s'est retrouvée en écho dans ces discours centrés sur l'élève et la question identitaire (associée à la culture et à la langue française). Les valeurs républicaines de la France ont trouvé leur place dans des discours centrés sur l'institution scolaire qui porte le citoyen en projet. Transversalement aux trois cas étudiés, les discours officiels sur l'éducation aux médias semblent alors avoir été instrumentalisés par des politiques porteuses de valeurs dont l'école a une responsabilité affirmée de transmission. Ces injonctions faites aux acteurs de l'éducation aux médias ne sont pas toujours lisibles en première lecture de ces discours, et la distance critique demandée aux élèves est à valoriser également auprès 
des enseignants qui pourraient se faire l'écho de cette idéologie à travers leurs propres engagements discursifs.

\section{BIBLIOGRAPHIE}

Anderson, James A. 1980. " The theoretical lineage of critical viewing curricula », Journal of Communication 30-3, 64-71

Barats, Christine. 2007. « Pour le prix d'un café par jour. Une analyse des logiques d'institutionnalisation des TIC par le ministère de l'Éducation nationale dans l'enseignement supérieur français depuis 1988 », Communication 25-2, 148-184

Bardin, Laurence. 2013. «L'analyse de contenu et de la forme des communications » (Paris : PUF) Buschini Fabrice \& Serge Moscovici (éds). Les méthodes des sciences humaines (Paris : PUF) Blanchet, Alain \& Anne Gotman. 2005. L'enquête et ses méthodes : l'entretien (Paris : Colin) Bonnafous, Simone \& Maurice Tournier. 1995. « Analyse du discours, lexicométrie, communication et politique », Langages 117, 67-81

Boutin, Perrine. 2011. « Le rapport des tout-petits au $7^{\mathrm{e}}$ art : débat autour d'École et cinéma ", Actes du colloque international Enfance et cultures, en ligne :

www.enfanceetcultures.culture.gouv.fr/actes/boutin.pdf

Carlsson, Ulla, Samy Tayie, Geneviève Jacquinot-Delaunay \& José M. Pérez Tornero (éds). 2008. Empowerment through Media Education, an intercultural Dialogue (Europe : ICCYM, UNESCO, Dar Graphit, Mentor Association)

Cerisier, Jean-François. 2014. "On demande toujours des inventeurs et l'on cherche encore les innovateurs ", Distances et médiations des savoirs 8, en ligne : $\underline{\text { http://dms.revues.org/891 }}$

Charaudeau, Patrick \& Dominique Maingueneau (dir.). 2002. Dictionnaire d'analyse du discours (Paris : Seuil)

Clanet, Claude (éd). 1985. L'interculturel en éducation et en sciences humaines, vol. 1-2 (Toulouse : Éditions Université Le Mirail)

Dunscombe, Roger \& Marlène Loicq. 2012. «Éducation aux médias en Australie. L’expérience du Victoria » Jeunes \& Médias, les cahiers francophones de l'éducation aux médias 4, «L'éducation aux médias dans le monde » (Paris : Éditions Publibook Université)

Foucault, Michel. 1969. L'archéologie du savoir (Paris : Gallimard)

Frau-Meigs, Divina. 2011. Socialisation des jeunes et éducation aux médias (Paris : Érès, Ceméa)

Gonnet, Jacques. 1995. De l'actualité à l'école : pour des ateliers de démocratie (Paris : Colin)

Groupe de travail sur la réforme du curriculum (GTRC). 1997. Réaffirmer l'école, Ministère de l'Éducation, Gouvernement du Québec, Québec (« Rapport Inchauspé »)

Jehel, Sophie. 2000. Parents ou médias, qui éduque les préadolescents? Enquête sur leurs pratiques TV, jeux vidéos, radio, Internet (Toulouse : Érès, CEMEA) 
Karsenti, Thierry \& Stéphanie Demers. 2000. «L'étude de cas », Karsenti, Thierry \& Lorraine Savoie-Zajc (éds). Introduction à la recherche en éducation (Sherbrooke : Éditions du CPR)

La Borderie, René. 1997. Éducation à l'image et aux médias (Noisy-le-Grand : Nathan-pédagogie)

Landry Normand \& Matthier Bégin. 2016. « Le programme de formation de l'école québécoise », Normand, Landry \& Anne-Sophie Lettelier. L'éducation aux médias à l'ère numérique. Entre fondations et renouvellement (Montréal : P. U. de Montréal), 83-98

Loicq, Marlène. 2012. «Éducation aux médias et problématiques interculturelles, question de méthodes », Questions de Communication 22, 285-300

Loicq, Marlène. 2011. Médias et interculturalité : l'éducation aux médias dans une perspective comparative internationale (Thèse de Doctorat)

Maingueneau, Dominique \& Frédéric Cossutta. 1995. «L'analyse des discours constituants ", Langages 117, 112-125

Masterman, Lenc \& François Mariet. 1994. L'éducation aux médias dans l'Europe des années 80 (Strasbourg : Éditions du Conseil de l'Europe).

Maurice, Marc. 1989. « Méthode comparative et analyse sociétale », Sociologie du travail 2, 175-191

Moeglin, Pierre. 2005. Outils et médias éducatifs. Une approche communicationnelle (Grenoble, P. U. de Grenoble)

Moirand, Sophie. 2007. Les discours de la presse quotidienne. Observer, analyser, comprendre (Paris : PUF)

Mucchielli, Alex (dir.). 2004. Dictionnaire des méthodes qualitatives en sciences humaines (Paris : Colin)

Oger, Claire. 2005. «L'analyse du discours institutionnel entre formations discursives et problématiques socio-anthropologiques », Langage \& Société 114, 113-128

Oger, Claire \& Caroline Ollivier-Yaniv. 2006. « Conjurer le désordre discursif. Les procédés de « lissage » dans la fabrication du discours institutionnel », Mots 81, 63-77

Oger, Claire \& Caroline Ollivier-Yaniv, 2003, « Analyse du discours institutionnel et sociologie compréhensive : vers une anthropologie des discours institutionnels », Mots 71, 125-145

Paquelin, Dominique. 2009. L'appropriation des dispositifs numériques de formation. Du prescrit aux usages (Paris : L'Harmattan)

Peraya, Daniel. 2011. Un regard critique sur les concepts de médiatisation et médiation. Nouvelles pratiques, nouvelle modélisation, en ligne : http://w3.u-grenoble3.fr/les_enjeux/2008-supplement/ Peraya/index.php

Piette, Jacques. 1988. La filiation théorique entre les programmes d'éducation aux médias et les courants de la recherche sur la communication de masse (mémoire de maîtrise, Université de Sherbrooke)

Piette, Jacques. 1996. L'éducation aux médias et fonction critique (Montréal : l'Harmattan)

Salazar-Orvig, Anne. 2003. « Éléments de sémiologie discursive » Moscovici, Serge \& Fabrice Buschini (éds). Les méthodes en sciences humaines (Paris : PUF), 271-296

Serres, Alexandre \& Marlène Loicq. 2014. «L'EMI (Education aux médias et à l'information) : quelles convergences et divergences au sein d'une nouvelle 'éducation à...' ? " Actes du colloque international des 17, 18 et 19 novembre 2014, «Les éducations à... : un (des) levier(s) de transformation du système éducatif ? », Université de Rouen/ESPé de l'Académie de Rouen, https://halshs.archivesouvertes.fr/halshs-01183403 


\section{NOTES}

1. Depuis la Déclaration de Grünwald le 22 janvier 1982, l'UNESCO est à l'initiative de rencontres internationales visant à instituer les étapes et enjeux de l'éducation aux médias (voir agenda de Paris en 2007, Moscou en 2012, etc.).

2. Si ce n'est pas le propos de cet article, il est important de préciser qu'en dépit de l'usage des mêmes arguments pour son implantation à l'école (nécessité d'une alphabétisation au langage médiatique structurant de nos sociétés modernes), ces expressions renvoient à deux champs distincts sur le plan historique et épistémologique (information literacy et media literacy Serres et Loicq 2015).

3. L'entretien est ici utilisé comme instrument privilégié de l'exploration des faits dont la parole est le vecteur (Blanchet et Gotman 2005) et permet de reconstituer l'histoire à partir de ceux qui la font. En cela, nous avons pu « éviter que la clôture du corpus ne devienne un obstacle à une compréhension globale des pratiques institutionnelles » (Oger $2005: 114$ ).

4. Notre corpus s'est attaché plus précisément à analyser les politiques éducatives dans l'État du Victoria, celui-ci étant le plus avancé en matière d'éducation aux médias au moment de la collecte des données. Aujourd'hui, la généralisation du curriculum à l'ensemble des États australiens permet d'étendre nationalement les résultats obtenus.

5. Australian Teachers of Media, appellation la plus récente de ce qui fut, dès 1960, le Joint Committee for the study of film and TV.

6. Centre de ressources en éducation aux médias.

7. Centre de liaison de l'enseignement et des moyens d'information, devenu le Centre de liaison de l'enseignement et des "médias" d'information", puis très récemment le Centre de l'éducation aux médias et à l'information.

8. Dans les faits, c'est à la rentrée 1981 que le cours était officiellement donné comme discipline autonome dans les écoles victoriennes.

9. Il le fera en demandant de « renforcer l'éducation aux médias » alors même que celle-ci n'est préalablement pas mise en place dans les programmes et encore moins définie par les discours prescriptifs. Les discours prescriptifs renvoient alors la fonction définitoire aux discours programmatiques portés par le CLEMI.

10. Dans le cadre de cet article, le corpus s'étend jusqu'en 2011. Les discours officiels les plus récents sur l'EMI ne sont donc pas pris en compte mais mériteraient une analyse similaire. Ils devraient notamment faire l'objet d'un travail de comparaison avec des politiques éducatives du numérique dont il est également temps de questionner les logiques discursives.

11. Les acteurs de l'institutionnalisation de l'éducation aux médias en Australie, au Québec et en France ont été rencontrés plusieurs fois et amenés à dialoguer dans le cadre d'entretiens semidirectifs et lors d'échanges plus informels lors desquels les imaginaires se laissent plus aisément saisir dans la mise en parole des représentations.

12. Le corpus débute en 1993 car les programmes de 1986 étaient inaccessibles via les archives du VCAA.

13. Pour une description plus précise de la relation complexe entre l'éducation aux médias et les valeurs socio-historiques des cas étudiés, voir ma thèse de Doctorat Médias et interculturalité : l'éducation aux médias dans une perspective comparative internationale (2011), disponible en ligne.

14. Sont d'ailleurs distingués les médias de l'image, fugaces, et ceux de l'écrit, plus légitimes et dignes d'intégrer l'école, selon une approche élitiste des supports et des genres médiatiques. 


\section{RÉSUMÉS}

Dans une démarche à la fois diachronique et synchronique, ce texte fait état d'une analyse socioanthropologique des discours officiels sur l'éducation aux médias dans trois contextes distincts (Australie, Québec, France). La spécificité des instances énonciatives y est questionnée à travers une approche comparative internationale en vue de dévoiler le rapport que les énoncés entretiennent avec le contexte socio-culturel duquel ils émergent. Il apparaît que l'éducation aux médias est un domaine qui se structure et est institué par des discours militants, programmatiques et prescriptifs, portés par des instances institutionnelles (ministères de l'éducation) et légitimes (organismes sous tutelle ou indépendants). Cette énonciation à deux voix participe de la construction discursive du champ de l'éducation aux médias dans le contexte scolaire.

In a diachronic and synchronic approach, this text presents a socio-anthropological analysis of the official discourses on media education in three distinct contexts (Australia, Quebec, France). The specificity of the enunciation is questioned through an international comparative approach in order to reveal the relation that the statements maintain with the socio-cultural context from which they emerge. It appears that media education is an area that is structured and is instituted by militant, programmatic and prescriptive discourses, carried out by institutional (ministries of education) and legitimate authorities. This two-part utterance is part of the discursive construction of the field of media education in the school context.

\section{INDEX}

Mots-clés : analyse discursive, approche comparative, discours instituants, médias et éducation Keywords : comparative approach, discourse analysis, institutive discourses, media and education

\section{AUTEUR}

\section{MARLÈNE LOICQ}

Université Paris-Est, UPEC Espé - Céditec 